\title{
Gas Transport in Porous Media: Simulations and Experiments on Partially Densified Aerogels
}

\author{
Anwar Hasmy, Isabelle Beurroies, Daniel Bourret and Rémi Jullien \\ Laboratoire de Science des Matériaux Vitreux, Université Montpellier II, Place Eugène Bataillon, \\ 34095 Montpellier, France
}

(December 13, 2018)

\begin{abstract}
The experimental density dependence of gas (argon and nitrogen) permeability of partially densified silica aerogels in the Knudsen regime is quantitatively accounted for by a computer model. The model simulates both the structure of the sintered material and the random ballistic motion of a point particle inside its voids. The same model is also able to account for the density dependence of the specific pore surface as measured from nitrogen adsorption experiments.
\end{abstract}

PACS numbers: 05.60.+w, 66.30.-h, 61.43.-j, 47.55.Mh 
The relation between fluid transport properties in porous materials and their geometrical characteristics is the subject of great experimental and theoretical activities, expecially after the new insights brought by modern scaling ideas and computer modelling facilities [1.2]. In the case of gas transport in the Knudsen regime, where the molecular diffusion is dominated by collisions with the pore walls, it has been demonstrated that the diffusion properties strongly depend on the geometrical characteristics of the pore network [3]. Therefore, to properly account for any experimental results there is a need for a right modelization of the internal structure of the material on a mesoscopic scale.

In this letter we present a comparison between experimental data and numerical calculations for the gas permeability and the specific pore surface of partially densified silica aerogels. The original (non-densified) aerogel structure is modelized by the diffusion-limited cluster-cluster aggregation model [4] which has been recently shown to successfully explain small angle scattering experiments on these materials [5]. The densification process is described by using a numerical realisation of recent scaling ideas for sintering [6] and the gas diffusivity is calculated by allowing a point particle to move within the pores of the sintered structure along successive random straight lines.

To build the original (non sintered) aerogel structure, we have considered a three dimensional off-lattice cluster-cluster model [7,8] which has already been extensively described elsewhere [0]. A set of $N$ identical spherical particles of diameter $a_{0}$ are initially randomly disposed (without overlap) in a cubic box of edge length $L a_{0}$, occupying a volume fraction, or dimension-less concentration of $c_{0}=(\pi / 6)\left(N / L^{3}\right)$. In the following the particles are assumed to have the density $\rho_{S}\left(=2.2 \mathrm{~g} / \mathrm{cm}^{3}\right)$ of pure silica so that the actual density is $c_{0} \rho_{S}$. Then, these particles are allowed to undergo a brownian diffusive motion and they irreversibly stick when they come at contact. Aggregates of particles are also able to diffuse together with the individual particles and to stick to particles or to other aggregates. In this process, the diffusion constant of the aggregates is considered to vary as the inverse of their radius of gyration and periodic boundary conditions are assumed at the box edges. When the concentration is sufficiently large (larger than a threshold value $c_{g}$ which tends 
to zero for infinite box size [5]), the final structure is a set of tangent spheres which can be described as a loose random packing of connected fractal aggregates, of fractal dimension $D \simeq 2$, whose mean size $\xi_{0}$ decreases as $c_{0}$ increases.

The sintering process, which consists in a strenghening of the internal structure accompanied by a gradual elimination of the pores, is here numerically simulated by following closely the reasoning of reference [6]. Introducing a sintering parameter $s$, which varies from zero (for the non sintered material) to a $c_{0}$-dependent upper limit (for the compact situation without hole), we first consider a $s$-dependent dressed structure in which each initial sphere of diameter $a_{0}$ is replaced by a sphere of same center but of larger diameter $a_{d}=a_{0}(1+s)$. Then the total volume $V_{d}(s)$ located inside the overlapping spheres is numerically calculated (avoiding multiple countings of overlaps).

The sintered structure is obtained from the dressed structure after applying a proper homogeneous volume contraction which insures mass conservation. The length reduction factor $\beta(s)$ is given by:

$$
\beta(s)=\left(\frac{6 V_{d}(s)}{N \pi a_{0}^{3}}\right)^{\frac{1}{3}}=\left(\frac{c(s)}{c_{0}}\right)^{\frac{1}{3}}
$$

where

$$
c(s)=\frac{V_{d}(s)}{L^{3} a_{0}^{3}}
$$

is the volume fraction of the sintered structure.

In figure 1 we provide a typical example with $c_{0}=0.1136$. A cut through the initial structure is shown as well as at two different stages $(s=1$ and $s=2)$ of the sintering process. Although in the last stages (when $c(s)$ becomes close to unity) the shapes of the holes are probably not well reproduced (they are certainly less smooth than in reality), this numerical method gives a quite good variation of the lower cut-off $a$ as a function of the aerogel density $\rho=c(s) \rho_{S}$, as it can be obtained from low angle neutron scattering experiments on partially densified silica aerogels. A detailed presentation of this numerical procedure, comparison with other numerical methods and scaling predictions [6] and confrontation with experiments will be reported elsewhere [9]. 
Here, we would like to focus on another geometrical characteristics which is measurable by adsorption experiments, namely the specific pore surface area. Knowing that the surface of the dressed structure is simply equal to $2 d V_{d} / d a_{d}=\left(2 / a_{0}\right)\left(d V_{d}(s) / d s\right)$, the specific surface $\Sigma(s)$ of the sintered material can be obtained after correcting by the adequate scaling factor and dividing by the total mass:

$$
\Sigma(s)=\frac{2}{a_{0} \rho_{S}} \frac{1}{V_{d}(s)} \frac{d V_{d}}{d s} \beta(s)=\frac{2}{a_{0} \rho_{S}} \frac{d \log c(s)}{d s} \beta(s)
$$

Starting from a given non-sintered structure, characterized by $L$ and $c_{0}$, one can calculate $c(s)$ (through $V_{d}(s)$ ) as well as its derivative, and, using formula (3), one can calculate the specific pore surface area as a function of $s$. Some results for the variation of the dimensionless quantity $a_{0} \rho_{S} \Sigma(s)$ as a function of $c(s)$ are reported on the log-log plots of figure 2 (dashed curves). One has checked that, for $s=0$, one recovers the exact result $a_{0} \rho_{S} \Sigma(0)=6$ (horizontal straight line shown in the figure), which is due to the fact that in that case the structure is made of identical non overlapping spheres, each of surface area $\pi a_{0}^{2}$ and mass $\rho_{S} \frac{\pi}{6} a_{0}^{3}$. Here the results have been obtained with $L=40.4$ and they result from an average over 20 realisations of the aerogel structure for each $c_{0}$ value. Here and in the following we have checked that the results are almost independent on $L$. It should be noticed that the scaling predicted in ref. [6] (a log-log slope of $D / 3(3-D) \simeq-0.7$ ) is here only observed in a quite small intermediate-c region indicating that corrections to scaling are important both at low $c\left(c \sim c_{0}\right)$ and at large $c(c \sim 1)$. Obviously $\Sigma$ tends to zero as $c$ tends to one.

To calculate the gas permeability $K(s)$, which is the ratio between a gas flux through the sintered material and the applied pressure gradient, we have used the fact that it is simply related to the gas diffusion constant $D_{s}(s)$ inside the pores by:

$$
K(s)=(1-c(s)) D_{s}(s)
$$

Introducing the gas diffusion constant inside the pores of the dressed structure $D_{d}(s)=$ $\beta(s)^{2} D_{s}(s)$ this formula also writes: 


$$
K(s)=\frac{1-c(s)}{\beta(s)^{2}} D_{d}(s)
$$

Since the experimental situation that we would like to describe correspond to low pressures and small gas particles (smaller than silica particles), the diffusion constant $D_{d}(s)$ is related to the Knudsen motion of a point particle inside the pores of the dressed structure. Given the dressed structure, characterized by $L, c_{0}$ and $s$, we have indirectly computed $D_{d}(s)$ by allowing a hard sphere of diameter $s a_{0}$ to perform a Knudsen motion inside the voids of the undressed (non sintered) structure made of spheres of diameter $a_{0}$. As already noticed [10], this is strictly equivalent to the original problem.

Initially a sphere of diameter $s a_{0}$ is released at a random point under the condition that it should not overlap any other sphere (when $s$ and/or $c_{0}$ are too large, many trials are necessary) and a random direction (uniformly distributed in space) is selected. Then the moving sphere is allowed to follow a random straight line motion in this direction until it collides a sphere of diameter $a_{0}$. Immediately after collision a new random direction is selected (in a half space) according to the so-called Knudsen cosine law [3,11], i. e. such that its probability distribution is proportional to $\cos \theta d^{2} \Omega$ where $\theta$ is the angle between this direction and the normal (center-to-center direction) and where $d^{2} \Omega=\sin \theta d \theta d \phi$ is the elementary solid angle. In practice $\theta$ is chosen such that $\cos ^{2} \theta$ is uniformly distributed between zero and one while the azimutal angle is chosen uniformly between 0 and $2 \pi$. It has been shown that this is essential to recover the equilibrium Boltzmann statistics and the right transport coefficients [3, 11]. Then, after a large number $N_{c}$ of collisions and using the periodic boundary conditions, one calculates the end-to-end square displacement $\ell^{2}$ of the diffusing sphere center as well as the total legth $\Lambda$ of its trajectory, which is the sum of the lengths of the successive segments. This allows us to calculate $D_{d}(s)$ by the well-known formula:

$$
D_{d}(s)=\frac{1}{6} v \frac{\ell^{2}}{\Lambda}
$$

where $v=\sqrt{8 k T / \pi m}$ is the mean molecular velocity of the gas particle of mass $m$ at temperature $T$. Reporting in formula (5), one finally gets: 


$$
K(s)=\frac{1}{6} v \frac{1-c(s)}{\beta(s)^{2}} \frac{\ell^{2}}{\Lambda}
$$

Some results for the variation with $c(s)$ of the dimensionless quantity $K(s) / a_{0} v$ are reported in the log-log plot of figure 3. In such calculation we have taken $\Lambda \simeq 10 L$ and we have averaged over 10 realizations of the original structure as well as 60 trials for the initial position, for each value of $c_{0}$. As expected $K$ tends to zero as $c$ tends to one. The curves obtained for different $c_{0}$ are quite close to each other, showing that the density dependence of the permeability is not strongly sensitive to the details of the structure (tangent or overlaping spheres). However we have shown above (see figure 2) that considering overlaping spheres to simulate the sintering is essential to account for a decrease of the specific pore surface with increasing densities.

Our simulation also allows us to investigate the statistics of the chords, which are the segments between successive collisions. It is known that the chord length distribution enters many properties of a porous material [3, 12]. In particular the mean chord length $\langle\ell>$ is directly related to the specific pore surface via a well known mathematical theorem [13]. In our simulation the mean chord length of the dressed structure can be simply obtained by $<\ell>=\frac{\Lambda}{N_{c}}$. Then going back to the sintered structure and using the theorem, we can derive the following alternative expression for $\Sigma(s)$ :

$$
\Sigma(s)=4 \frac{1-c(s)}{c(s)} \frac{\beta(s)}{\rho_{S}<\ell>}
$$

We have reported $a_{0} \rho_{S} \Sigma$ as a function of $c(s)$, calculated from $<\ell>$ with the same $c_{0}$ values as before on the same plot of figure 2. While the overall $c$-dependence is qualitatively the same, we observe some discrepancies compared to the preceeding calculation which increase for larger $c_{0}$. Even if we have checked that the discrepancies do not result from introducing an upper cut off to the chord length at $\Lambda$, the numerical uncertainties are certainly larger in the second calculation. It may also happen that, for larger concentrations, the presence of closed pores may affect differently the two calculations (closed pore surface is fully considered in the first calculation). Moreover, the theorem holds for a globally isotropic pore network 
and, even if all the cluster-cluster growth conditions are isotropic, the resulting gel may be not since it is known that the cluster-cluster aggregates are intrinsically anisotropic [14].

The gas permeability experiments on partially densified aerogels have been performed using a newly designed apparatus which allows to measures simultaneously the gas flux through a cylindrical sample and the gas pressure difference between the two edges. Technical details will be given elsewhere [15]. Here we report on experiments done with a series of aerogels which have been prepared under neutral conditions and for which pore surface area data have been previously measured by nitrogen adsorption experiments [6]. Before sintering, their density is known to be equal to $\rho_{0}=0.25 \mathrm{~g} / \mathrm{cm}^{3}$. Two series of experiments have been done with different gas, nitrogen and argon. The experimental results for both the ratio $K / v$ and the pore surface area $\Sigma$ are reported in figure 4 . As expected, one observes that, after dividing by the gas velocity, the data are superimposed. In both cases the symbols correspond to the experimental data and the full lines correspond to a fit with our computer model using $c_{0}=\rho_{0} / \rho_{S}=0.1136, L=40.4$, and $a_{0}=70 \AA$. It is remarkable that, with only one adjustable parameter $\left(a_{0}\right)$, we are able to fit quite well the two quantities simultaneously (formula (3) has been used for $\Sigma$ ). This gives a good confidence in the pertinence of our model. Although of the right order of magnitude, the value chosen for $a_{0}$ is larger than other previous estimates for the mean particle diameter of neutral aerogels [16]. Here, we have considered a set of monodisperse spheres while it is well known that the size polydispersity of individual particles is very large in neutral aerogels [16]. As already noticed for small angle scattering experiments [17], the large balls dominate any scattering process and they also have a greater contribution to the internal pore surface area. This could explain why we are obliged to consider here an effective particle diameter larger than the simple mean. Also, counting the closed pores (which are consisered in the fit) could also increase the effective $a_{0}$.

In conclusion, using a computer model which simulates both the formation and densification process of aerogels we have been able to quantitatively account for the density dependence of the gas permeability and pore surface area. In the future we will pursue the 
numerical simulations of aerogels sintering, and we will extend the present model, introducing polydispersity and analysing more extensively the chord length distribution. We have also in project to simulate the brownian diffusion regime in connection with the transport properties of fluids inside aerogels.

We would like to acknowledge discussions with R. Sempere, T. Woignier, J. Phalippou, M. Foret and J. Pelous. One of us (A. H.) would like to acknowledge support from CONICIT(Venezuela). Laboratoire de Sciences des Matériaux Vitreux is Unité Associée au CNRS No. 1119. 


\section{REFERENCES}

[1] M. B. Isichenko, Rev. of Mod. Phys., 64, 961, 1992.

[2] M. Sahimi, Rev. of Mod. Phys. D., 65, 1393, 1993.

[3] P. Levitz, J. Phys. Chem., 97, 3813, 1993.

[4] R. Jullien, Aggregation and Fractal Aggregates, World Scientific, Singapore, 1987.

[5] A. Hasmy, E. Anglaret, M. Foret, J. Pelous and R. Jullien, Phys. Rev. B, to be published on 1st september 1994.

[6] R. Sempere, D. Bourret, T. Woignier, J. Phalippou and R. Jullien, Phys. Rev. Lett., 71, 3307, 1993.

[7] P. Meakin, Phys. Rev. Letters, 51, 1119, 1983.

[8] M. Kolb, R. Botet and R. Jullien, Phys. Rev. Letters, 51, 1123, 1983.

[9] N. Olivi-Tran, A. Hasmy, G. Cohen-Solal and R. Jullien, in preparation

[10] S. Torquato, J. Chem. Phys., 95, 2838, 1991.

[11] J. P. Walleau, D. J. Diestler, J. H. Cushman, M. Schoen, A. W. Hertzner and M. E. Riley, J. Chem. Phys., 95, 6194, 1991.

[12] P. Levitz and D. Tchoubar, J. de Physique I (France), 2, 771, 1992.

[13] F. A. Dullien, Porous Media: Fluid transport and Pore Structure, Academic press, New York, 1976.

[14] R. Botet and R. Jullien, J. Phys. A, 19, L907, 1986.

[15] I. Beurroies, D. Bourret, R. Sempere, J. Phalippou and T. Woignier, preprint.

[16] R. Vacher, T. Woignier, J. Pelous and E. Courtens, Phys. Rev. B, 37, 6500, 1988.

[17] A. Hasmy, R. Vacher and R. Jullien, Phys. Rev. B, rapid communication, to be published 
on 1st july 1994. 


\section{FIGURES}

FIG. 1. Cut of the simulated aerogel structure at three stages of sintering, $s=0,1$ and 2, where $c(s)=0.1136,0.54,0.88$, repectively. Here and in the other figures the box size is $L=40.4$.

FIG. 2. Log-log plot of $a_{0} \rho_{S} \Sigma$ versus $c$. Dotted, dashed and dot-dashed curves correspond to formula (3) with $c_{0}=0.05,0.1136$ and 0.2 , respectively. The horizontal line correspond to the

exact result $a_{0} \rho_{S} \Sigma=6$ for $c=c_{0}$. Triangles, diamonds and squares correspond to formula (8) with $c_{0}=0.05,0.1136$ and 0.2 , respectively.

FIG. 3. Log-log plot of $K / a_{0} v$ versus $c$. Triangles, diamonds and squares correspond to $c_{0}=0.05,0.1136$ and 0.2 , respectively

FIG. 4. Fits of both $K / v$ and $\Sigma$ for neutral aerogels. In the case of $K / v$ open squares and diamonds correspond to argon and nitrogen, respectively. The lines are the fits with $L=40.4$, $c_{0}=0.1136$ and $a_{0}=70 \AA$. 
fig $1 \mathrm{a}$

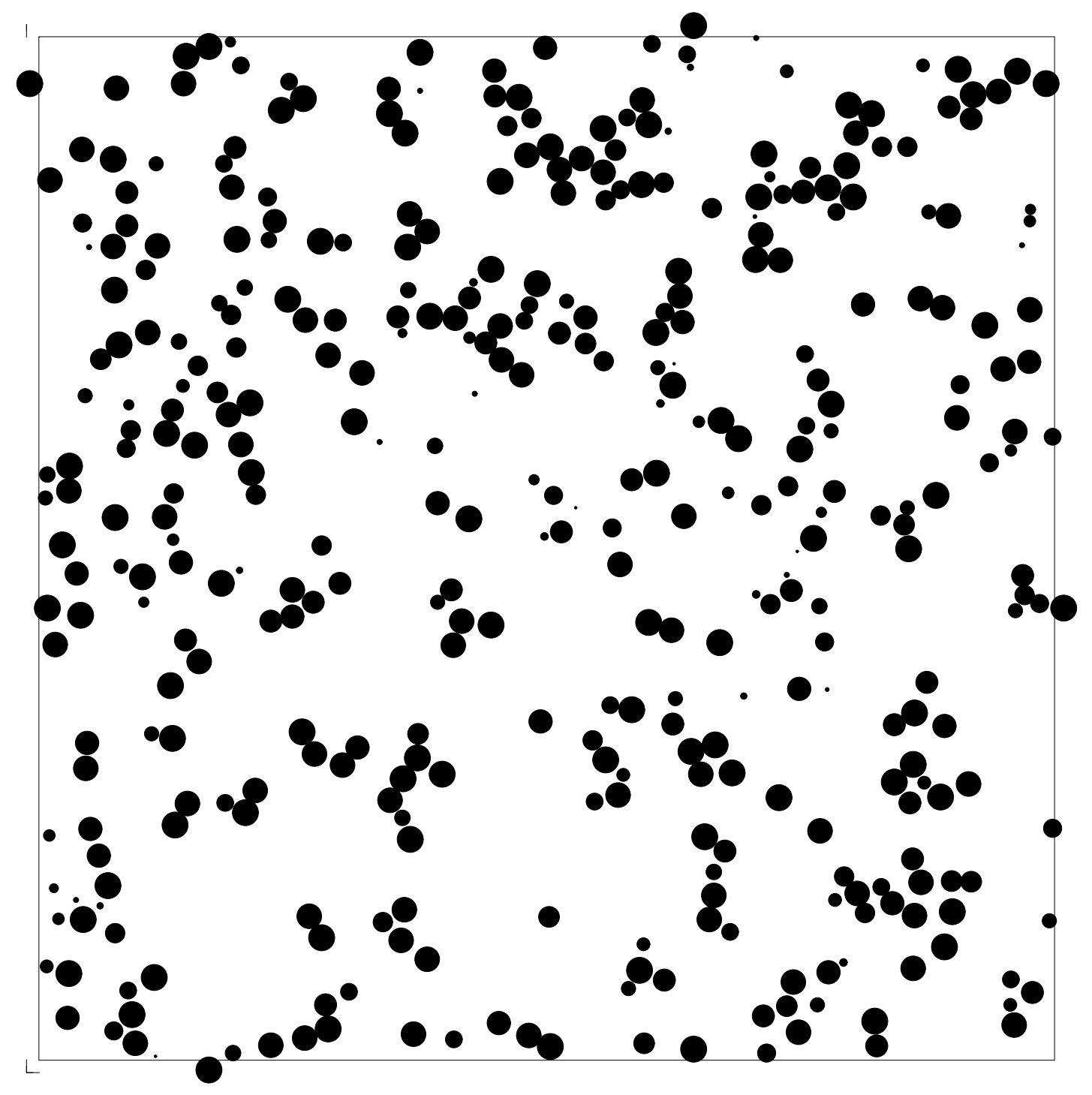


fig $1 b$

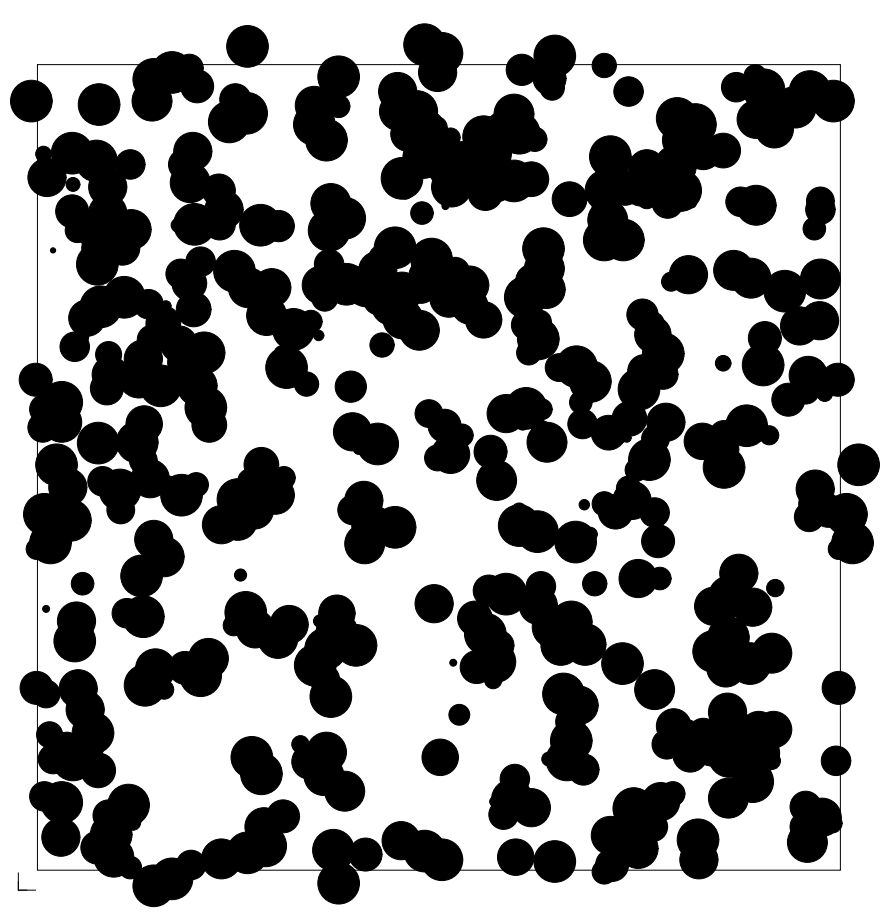


fig $1 \mathrm{c}$

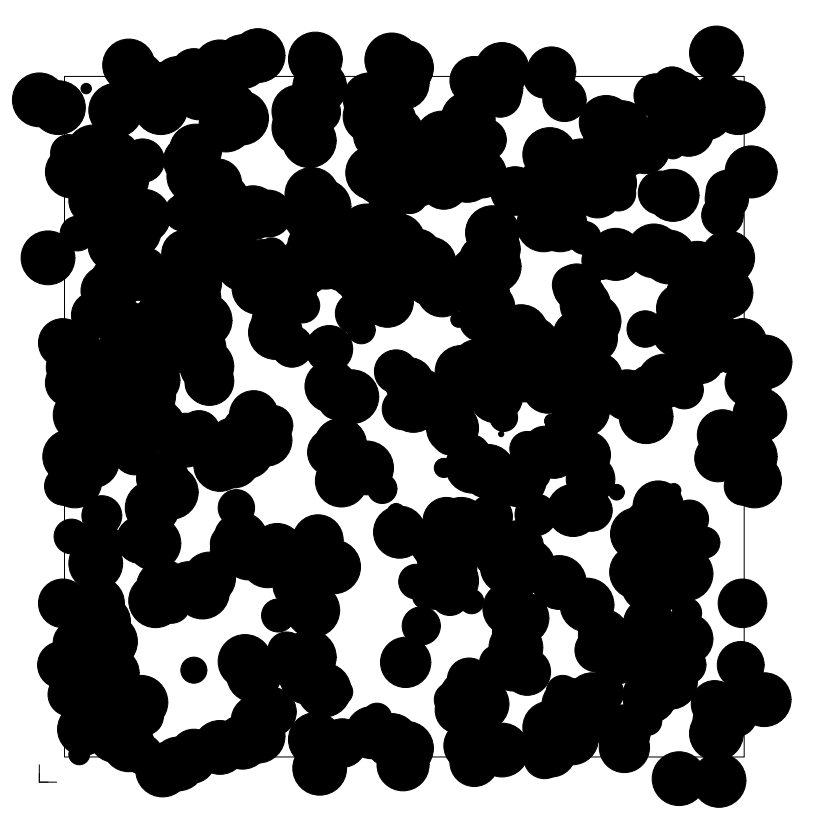




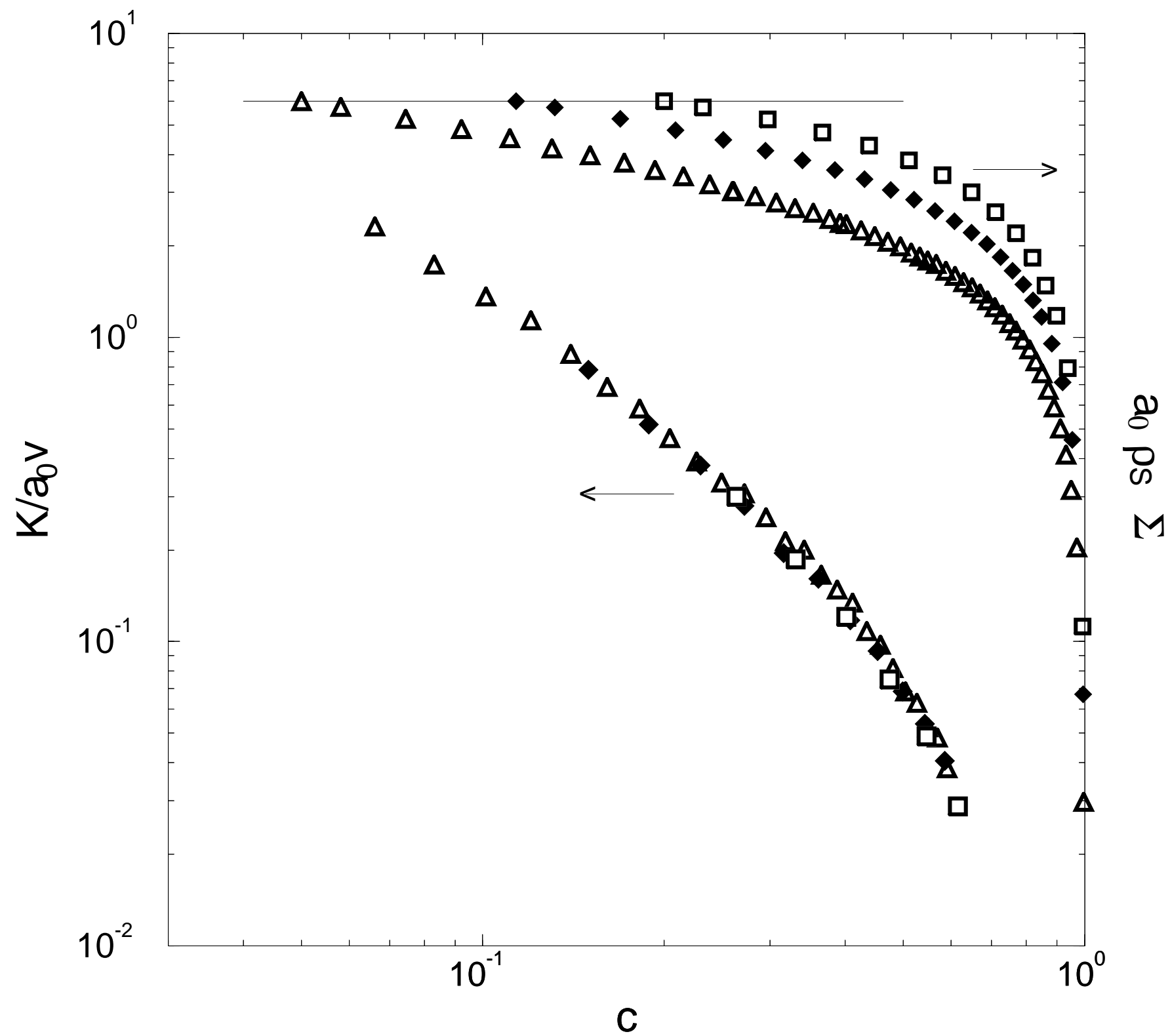




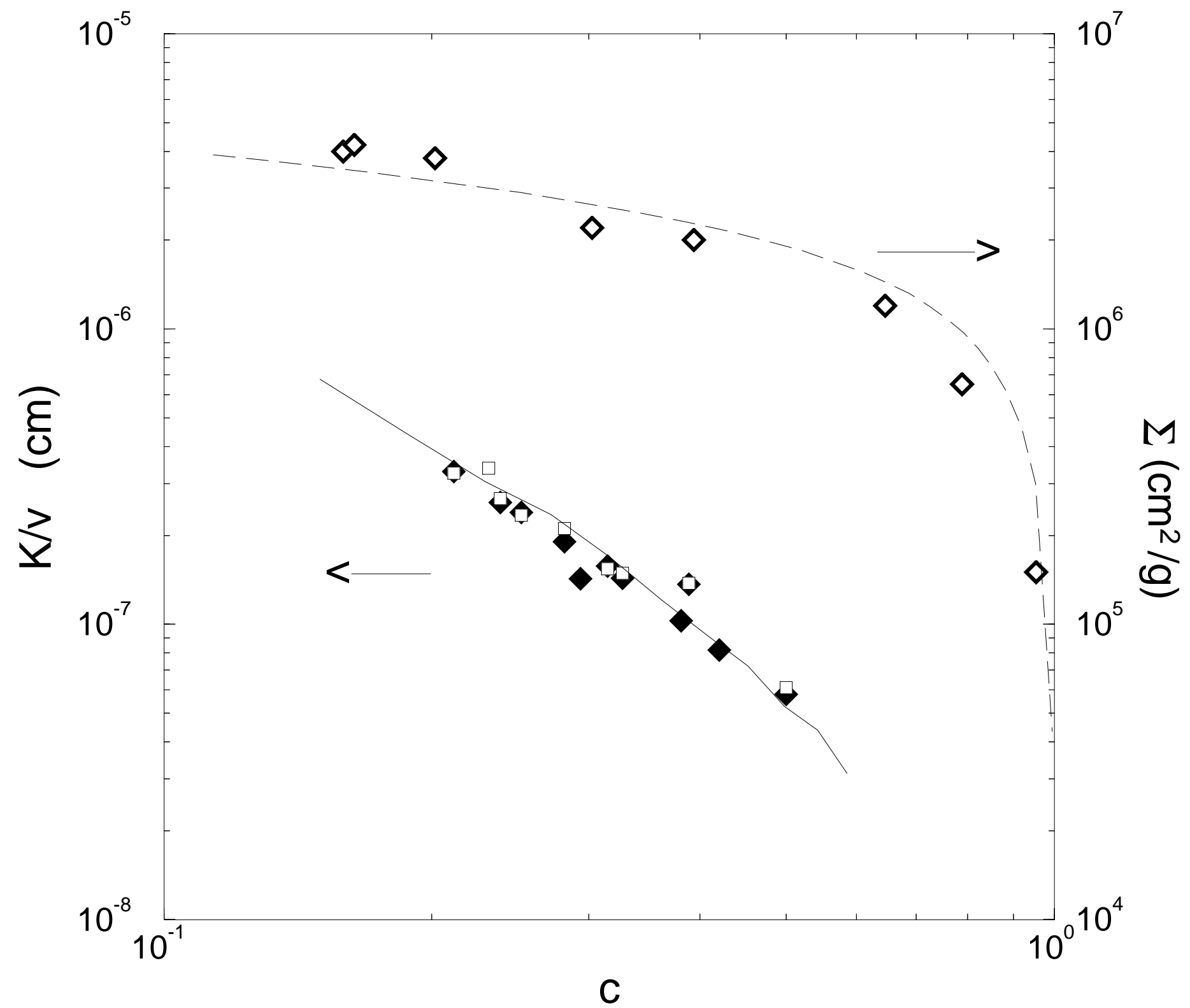

